\title{
Relativistic effect on atomic displacement damage for two-body inducing discrete reactions
}

\author{
Shengli Chen ${ }^{1,2, *}$, David Bernard ${ }^{1}$, and Cyrille De Saint Jean ${ }^{1}$ \\ ${ }^{1}$ CEA, Cadarache, DEN/DER/SPRC/LEPh, 13108 Saint Paul Les Durance, France \\ ${ }^{2}$ I-MEP2, University Grenoble Alpes, 38402 Saint Martin d'Heres, France
}

\begin{abstract}
The relativistic effect on two-body discrete reaction inducing atomic recoil energy and the sequent damage energy is studied for ${ }^{6} \mathrm{Li},{ }^{56} \mathrm{Fe},{ }^{184} \mathrm{~W}$, and ${ }^{238} \mathrm{U}$. The relativistic correction is within $1 \%$ if incident neutron energy is below $20 \mathrm{MeV}$. For incident neutron energy up to $200 \mathrm{MeV}$ or even $800 \mathrm{MeV}$, the relativistic effect should be taken into account for treating two-body kinematics. The relativistic correction is about $0.05 E_{\text {in }} / \mathrm{MeV} \%$ for neutron elastic scattering for nuclei from ${ }^{56} \mathrm{Fe}$ to ${ }^{238} \mathrm{U}$ and smaller for $(\mathrm{n}, \alpha)$ and $(\mathrm{n}, \mathrm{t})$ reactions. Analyses on damage energy show that the relativistic corrections are generally within $2 \%$ for incident neutron below $200 \mathrm{MeV}$ for nuclei lighter than ${ }^{56} \mathrm{Fe}$ because of the "saturation" of damage energy. However, the current damage theory cannot be applied for Primary Knock-on Atom (PKA) energy higher than $24.9 A_{R} Z_{R}^{4 / 3}$ $\mathrm{keV}$, which is 10 times lower than the maximum PKA energy for D+T fusion neutron elastic scattering of ${ }^{6} \mathrm{Li}$.
\end{abstract}

\section{Introduction}

Irradiation damage changes the properties of nuclear materials, such as the reduction of ductility, primary radiation hardening, irradiation creep and growth, and swelling of materials [1]. The irradiation damage is conventionally quantified by the number of Displacement per Atom (DPA) in materials. In the past decades, many models have been developed to compute the DPA using the kinetic energy of the Primary Knock-on Atom (PKA) as a major parameter. Our recent studies show the importance of relativistic effect on PKA energy for high energy incident neutron-induced reactions $[2,3]$. Because the relativistic correction on discrete reactions depends on target nucleus [3], the present work focuses on the relativistic effect on neutron-induced discrete two-body reactions of ${ }^{6} \mathrm{Li},{ }^{56} \mathrm{Fe}$, ${ }^{184} \mathrm{~W}$, and ${ }^{238} \mathrm{U}$ with nuclear data from JEFF-3.1.1 [4].

\section{Damage energy}

Due to electronic excitation and ionization during the displacement cascade, damage energy $\left(E_{a}\right)$ defines the available energy for atomic displacement in materials. Fig. 1 shows damage energy and partition function (i.e. $\left.E_{a} / E_{P K A}\right)$ versus PKA energy. This section shows further discussions on damage energy theory, including the upper limit, asymptotic value, and maximum damage energy. The basic summary of the damage theory and the notation used in this section can be found in Ref. [3]. The conclusions are used in the analyses on the relativistic effect in this paper.

*e-mail: shengli.chen@cea.fr
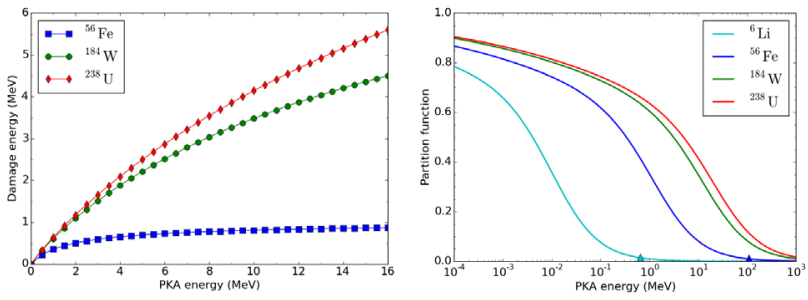

Figure 1. Damage energy and partition function vs PKA energy

Table 1. Upper limits of PKA energy (in MeV) for the validation of damage energy or NRT-DPA formula

\begin{tabular}{lllllll}
\hline PKA & ${ }^{3} \mathrm{H}$ & ${ }^{4} \mathrm{He}$ & ${ }^{6} \mathrm{Li}$ & ${ }^{56} \mathrm{Fe}$ & ${ }^{184} \mathrm{~W}$ & ${ }^{238} \mathrm{U}$ \\
\hline Limit & 0.0747 & 0.251 & 0.646 & 107 & 1423 & 2461 \\
\hline
\end{tabular}

Upper limit of the damage theory Because Lindhard's equation is based on the assumption that PKA energy is lower than $24.9 A_{R} Z_{R}^{4 / 3} \mathrm{keV}$ (the subscript $R$ represents recoil nucleus) [7], the current standard NRT-DPA formula [8] is valid only for $E_{P K A}<24.9 A_{R} Z_{R}^{4 / 3} \mathrm{keV}$. Table 1 shows the upper limits of the validation of damage energy or NRT-DPA formula for different PKAs. The corresponding values are indicated by triangles in Fig. 1(b). It is noticeable that the upper limit is $646 \mathrm{keV}$ for ${ }^{6} \mathrm{Li} \mathrm{PKA}$, while the maximum PKA energy is about $6.9 \mathrm{MeV}$ for $\mathrm{D}+\mathrm{T}$ fusion neutron $(14.1 \mathrm{MeV})$ elastic scattering.

Asymptotic value of damage energy Neglecting the limit range of damage energy, one can obtain the asymptotic value (in $\mathrm{eV}$ ) as:

$$
E_{a}^{\infty}=\frac{E_{L}}{k_{L}}=\frac{387.44 Z_{R}^{1 / 3} Z^{1 / 2}\left(Z_{R}^{2 / 3}+Z^{2 / 3}\right)^{5 / 4} A_{R}^{3 / 2}}{A^{1 / 2}\left(A_{R}+A\right)^{1 / 2}}
$$


Table 2. Monotonicity of the upper limit of damage energy

\begin{tabular}{lcccc}
\hline Variable & $Z_{R}$ & $A_{R}$ & $Z$ & $A$ \\
\hline Monotonicity & $\nearrow$ & $\nearrow$ & $\nearrow$ & $\searrow$ \\
\hline
\end{tabular}

The monotonicity of $E_{a}^{\infty}$ versus different variables are summarized in Table 2. The conclusions on $Z_{R}, Z$ and $A$ are evident from Eq. 1, while that on $A_{R}$ is determined by calculating the partial derivative. In the case that $A_{R}=A$, Eq. 1 implicates that $E_{a}^{\infty}$ increases with $A$.

Maximum damage energy Using the maximum PKA energy $24.9 A_{R} Z_{R}^{4 / 3} \mathrm{keV}$, the maximum damage energy can be directly obtained. However, in order to obtain a simple relationship between the maximum damage energy and the asymptotic value, we assume that $A_{R}=2 Z_{R}$ and $A=2 Z$, so that:

$$
k_{L}=\frac{0.0793\left(Z_{R}+Z\right)^{1 / 2}}{\sqrt{2}\left(Z_{R}^{2 / 3}+Z^{2 / 3}\right)^{3 / 4}}
$$

The monotonic analysis shows $0.04715 \leq k_{L}<0.05607$. On the other hand, $\epsilon_{\max }=286 A / Z \approx 600$. Supposing $k_{L} \approx 0.05$, one obtains $E_{a}^{\max } \approx 0.88 E_{a}^{\infty}$.

In the case where $Z_{R} \approx Z$ and $A_{R} \approx A$, the asymptotic and maximum damage energies (in $\mathrm{eV}$ ) are:

$$
\begin{aligned}
E_{a}^{\infty} & =652 Z^{5 / 3} A^{1 / 2} \\
E_{a}^{\max } & =573 Z^{5 / 3} A^{1 / 2}
\end{aligned}
$$

As shown in Fig. 1, it is noteworthy that the damage energy is almost "saturated" for relatively high PKA energy (more exactly, for high $\epsilon$ value). For ${ }^{56} \mathrm{Fe}$ PKA in ${ }^{56} \mathrm{Fe}, 4 \mathrm{MeV}$ PKA energy can have almost the "saturation" value of damage energy.

\section{Results and discussion}

\subsection{Relativistic effect on recoil energy}

Fig. 2 (Fig. 3 resp.) shows the recoil energies calculated with relativistic kinematics and the classical collisions for $20 \mathrm{MeV}$ (200 MeV resp.) neutron scattering reactions of ${ }^{6} \mathrm{Li},{ }^{56} \mathrm{Fe},{ }^{184} \mathrm{~W}$, and ${ }^{238} \mathrm{U}$. Fig. 4 shows the results for charged particle emission reactions of ${ }^{6} \mathrm{Li}$ and ${ }^{184} \mathrm{~W}$. The results of discrete $(\mathrm{n}, \mathrm{p})$ and $(\mathrm{n}, \alpha)$ reactions of ${ }^{56} \mathrm{Fe}$ are shown in Ref. [3]. No (n,p) and $(n, \alpha)$ is given for ${ }^{238} \mathrm{U}$ in JEFF-3.1.1 [4].

Figs. 2-4 show that the relativistic correction depends on reaction type, target nucleus, excitation level, emission angle, and incident neutron energy. However, for neutron energy below $20 \mathrm{MeV}$, which is the case for both fission and fusion reactors, the relativistic corrections are globally within $1 \%$ for the target from ${ }^{6} \mathrm{Li}$ to ${ }^{238} \mathrm{U}$.

For $200 \mathrm{MeV}$ incident energy, the dependence of the relativistic correction on excitation level is weak because the excitation energies (several $\mathrm{MeV}$ ) are quite negligible compared with the incident energy. The relativistic corrections are almost $10 \%$ for discrete neutron scattering and $(\mathrm{n}, \mathrm{p})$ reactions of nuclei from ${ }^{6} \mathrm{Li}$ to ${ }^{238} \mathrm{U}$. For $(\mathrm{n}, \alpha)$ reactions, the relativistic corrections are [-6\%, 5\%] for ${ }^{56} \mathrm{Fe}$ [3] and ${ }^{184} \mathrm{~W}$. For $200 \mathrm{MeV}$ neutron-induced $(\mathrm{n}, \mathrm{t})$ reaction of ${ }^{6} \mathrm{Li}$, the corrections are in $[-6 \%, 2 \%]$.

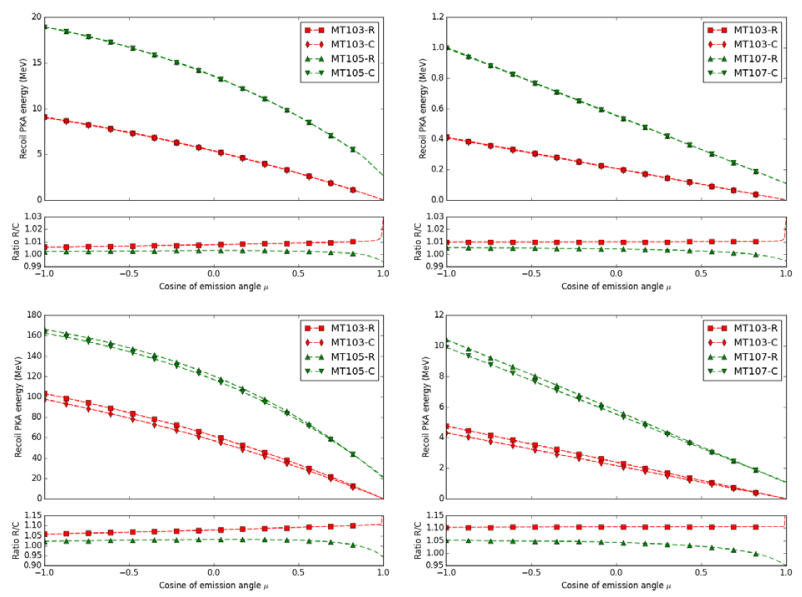

Figure 4. Charged particle emission reaction-induced PKA energy $\left(20 \mathrm{MeV} \mathrm{n}+{ }^{6} \mathrm{Li},{ }^{184} \mathrm{~W} ; 200 \mathrm{MeV} n+{ }^{6} \mathrm{Li},{ }^{184} \mathrm{~W}\right)$

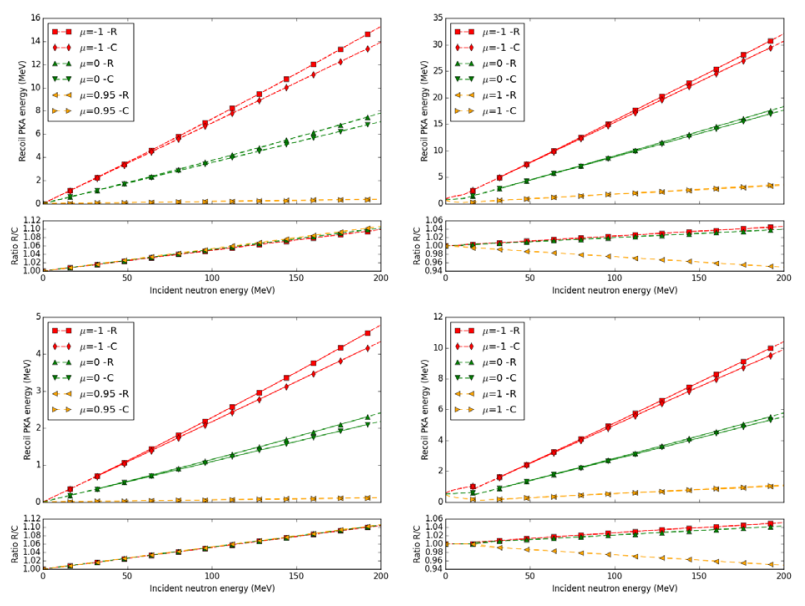

Figure 5. PKA energy versus incident energy for different emission angles $\left({ }^{56} \mathrm{Fe}\left(\mathrm{n}, \mathrm{n}^{\prime}\right),(\mathrm{n}, \alpha) ;{ }^{184} \mathrm{~W}\left(\mathrm{n}, \mathrm{n}^{\prime}\right),(\mathrm{n}, \alpha)\right)$
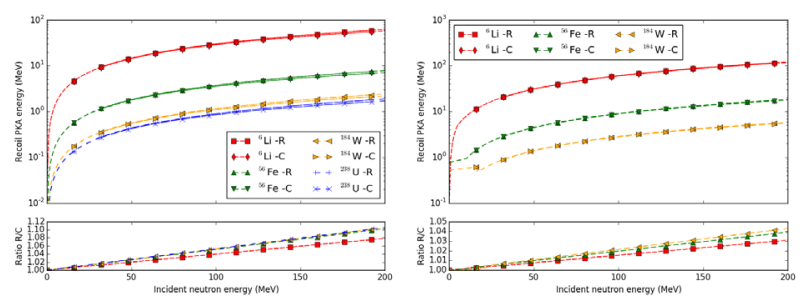

Figure 6. PKA energy versus incident energy for $\mu=0$ ((n,n'), $(\mathrm{n}, \alpha)\left((\mathrm{n}, \mathrm{t})\right.$ for $\left.\left.{ }^{6} \mathrm{Li}\right)\right)$

Fig. 5 illustrates the relativistic corrections of PKA energy neutron elastic scattering $(n, n)$ and the $(n, \alpha)$ reaction with different emission angle versus incident energy. Fig. 6 shows the same results for various nuclei with $\mu=0$. Fig. 5 points out that the relativistic correction is not much sensitive to emission angle for elastic scattering with $\mu<1$ (the PKA energy at $\mu=1$ is null). However, the correction on (n, $\alpha)$-induced PKA energy depends on the emission angle. These two conclusions are in agreement with 

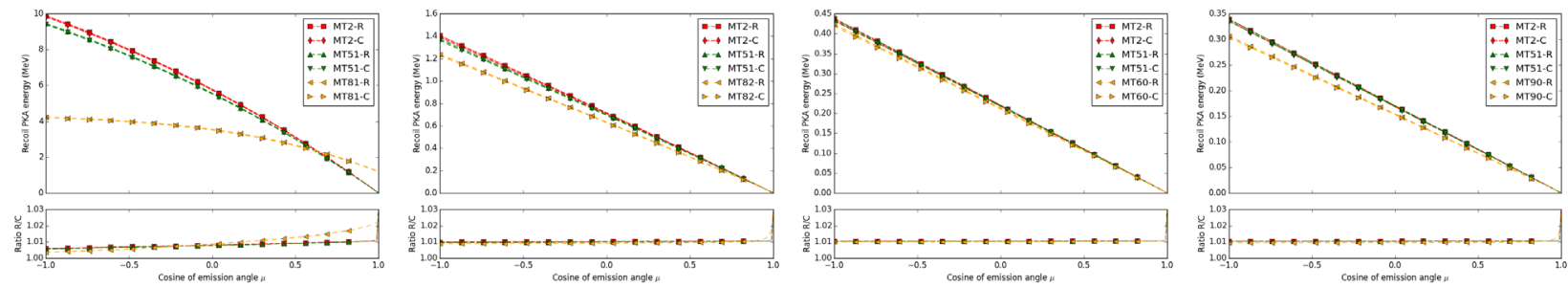

Figure 2. $20 \mathrm{MeV}$ neutron scattering-induced PKA energy calculated with relativistic (R) and classic (C) kinematics and the corresponding relativistic correction (R/C) with ${ }^{6} \mathrm{Li},{ }^{56} \mathrm{Fe},{ }^{184} \mathrm{~W}$, and ${ }^{238} \mathrm{U}$ targets
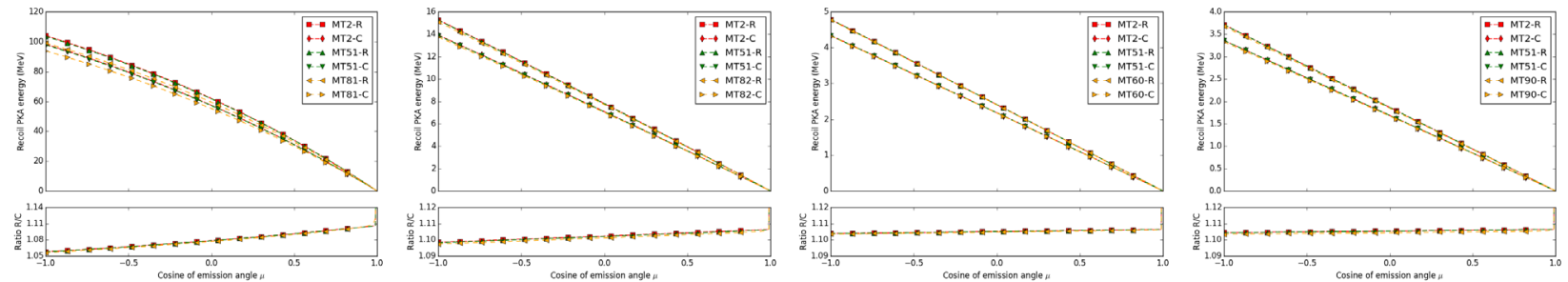

Figure 3. $200 \mathrm{MeV}$ neutron scattering-induced PKA energy calculated with relativistic (R) and classic (C) kinematics and the corresponding relativistic correction (R/C) with ${ }^{6} \mathrm{Li},{ }^{56} \mathrm{Fe},{ }^{184} \mathrm{~W}$, and ${ }^{238} \mathrm{U}$ targets

the results shown in Figs. 2-4. The R/C's for (n, $\alpha$ ) (and $(\mathrm{n}, \mathrm{t})$ for ${ }^{6} \mathrm{Li}$ ) are smaller than the ones for scattering reaction. The strange forms below $\sim 5 \mathrm{MeV}$ in Figs. 5 and 6 for charged particle emission reactions are due to the use of the Coulomb barrier energy as the minimum kinetic energy of the system.

Fig. 6 shows that the relativistic corrections have an almost linear relationship with incident energy. Except for ${ }^{6} \mathrm{Li}$, the relativistic correction on PKA from ${ }^{56} \mathrm{Fe}$ to ${ }^{238} \mathrm{U}$ with neutron elastic scattering is approximately:

$$
R / C-1(\%) \approx 0.05 E_{\text {in }} / \mathrm{MeV}
$$

where $E_{\text {in }}$ represents the incident neutron energy. Since the Center of Mass (CM) is not defined in the special relativity, the differential cross sections given in the $\mathrm{CM}$ frame cannot be directly used in relativistic kinematics. In this case, the simple corrections on recoil energy as Eq. 4 can be used to compute irradiation damage $[5,6]$.

\subsection{Relativistic effect on damage energy}

To evaluate the correction of relativistic effect on atomic displacement damage, the damage theory [7] is used in the present work. Fig. 7 shows the damage energy based on the relativistic kinematics and the classic mechanics for 20 $\mathrm{MeV}$ (upper figures) and $200 \mathrm{MeV}$ (lower figures) neutron scattering reactions of ${ }^{56} \mathrm{Fe}$ and ${ }^{238} \mathrm{U}$. The results about the damage energy of ${ }^{6} \mathrm{Li}$ are not shown because the PKA energies are already higher than the upper limit of damage theory (c.f. limits given in Table 1 and PKA energies shown in Figs. 2 and 3). Fig. 8 shows the same results for discrete (n,p) and (n, $\alpha$ ) reactions of ${ }^{56} \mathrm{Fe}$ and ${ }^{184} \mathrm{~W}$.

Figs. 7 and 8 show that the relativistic correction on damage energy is not sensitive to the excitation levels,
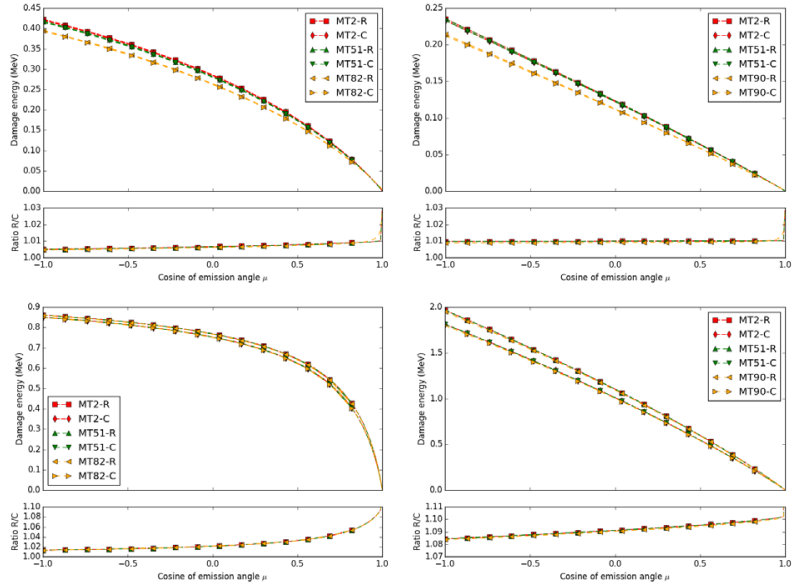

Figure 7. Damage energy for neutron scattering reactions (20 $\left.\mathrm{MeV} \mathrm{n}+{ }^{56} \mathrm{Fe},{ }^{238} \mathrm{U} ; 200 \mathrm{MeV} \mathrm{n}+{ }^{56} \mathrm{Fe},{ }^{238} \mathrm{U}\right)$

which is in agreement with the conclusion for PKA energy. However, due to the different atomic numbers and atomic masses, the relativistic effect on damage energy depends much on target nuclei, while that on PKA energy is not much sensitive to the targets. Therefore, the relativistic correction on damage energy should be treated for each specific target and each nuclear reaction.

Figs. 9 and 10 illustrate the damage energies corresponding to the PKA energies shown in Figs. 5 and 6. For $n+{ }^{56} \mathrm{Fe}$ reactions, because the damage energy is almost "saturated" at PKA energy above MeV energy (as shown in Fig. 1), the relativistic correction on damage energy is generally within $2 \%$ for neutron energy up to $200 \mathrm{MeV}$. The $\mathrm{R} / \mathrm{C}$ values are larger for $\mu>0$ (especially for $\mu=1$ ) due to the smaller PKA energies. Since Fig. 1 shows that 


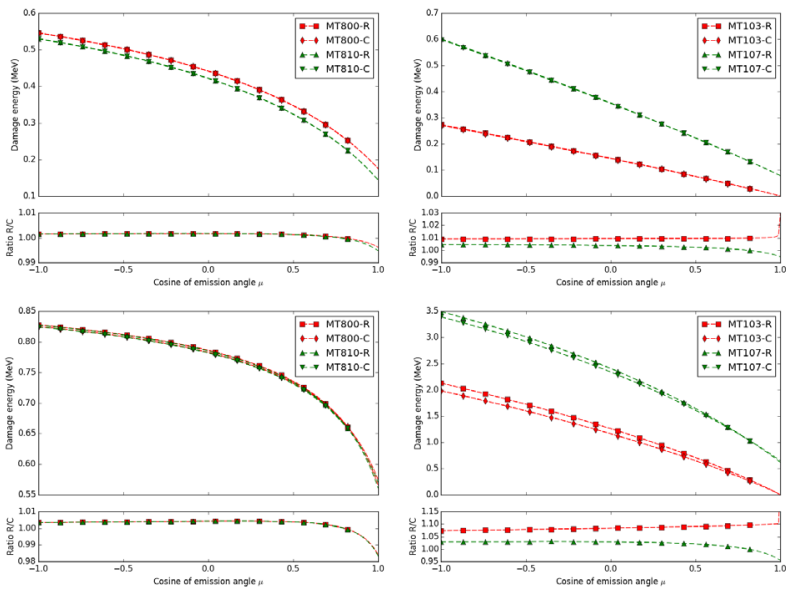

Figure 8. Damage energy for discrete $(\mathrm{n}, \mathrm{p})$ and $(\mathrm{n}, \alpha)$ reactions $\left(20 \mathrm{MeV} \mathrm{n}+{ }^{56} \mathrm{Fe},{ }^{184} \mathrm{~W} ; 200 \mathrm{MeV} \mathrm{n}+{ }^{56} \mathrm{Fe},{ }^{184} \mathrm{~W}\right)$
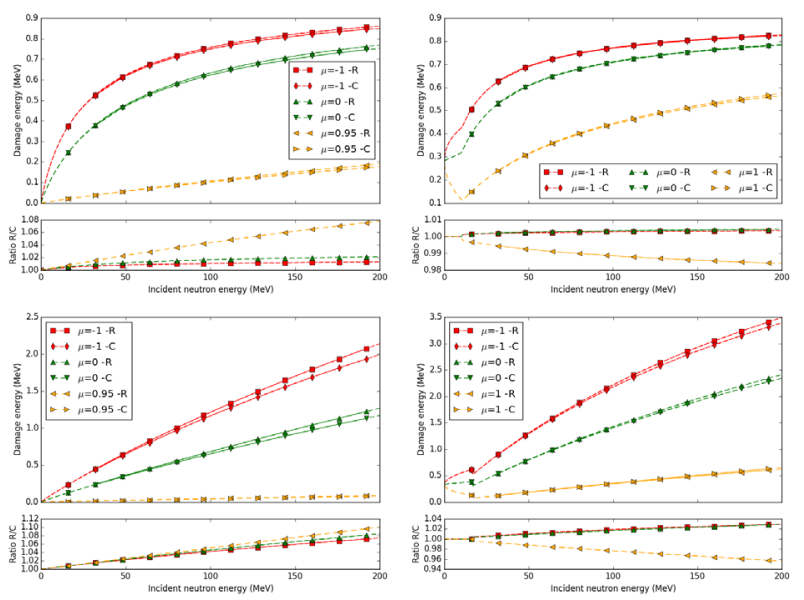

Figure 9. Damage energy versus incident energy for different emission angles $\left({ }^{56} \mathrm{Fe}\left(\mathrm{n}, \mathrm{n}^{\prime}\right),(\mathrm{n}, \alpha) ;{ }^{184} \mathrm{~W}\left(\mathrm{n}, \mathrm{n}^{\prime}\right),(\mathrm{n}, \alpha)\right)$
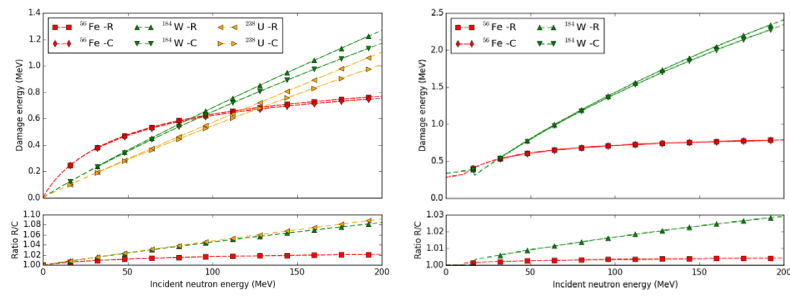

Figure 10. Damage energy versus incident energy for $\mu=0$ $\left(\left(\mathrm{n}, \mathrm{n}^{\prime}\right),(\mathrm{n}, \alpha)\right)$

the damage energy is still far away from the "saturation" for ${ }^{184} \mathrm{~W}$ and ${ }^{238} \mathrm{U}$, the corresponding relativistic effects are much more important than that of ${ }^{56} \mathrm{Fe}$.

\section{Conclusions}

The damage energy is widely used to compute the primary damage of materials via the international standard formula NRT-DPA. However, attention should be paid for light PKAs, of which the upper boundaries of damage theory can be lower than the corresponding maximum PKA energies in applications. For example, the upper limit of the Lindhard's damage theory is $646 \mathrm{keV}$ for ${ }^{6} \mathrm{Li} \mathrm{PKA}$, while the maximum PKA energy for $\mathrm{D}+\mathrm{T}$ fusion neutron elastic scattering is about $6.9 \mathrm{MeV}$. Moreover, for high PKA energy (e.g. above $5 \mathrm{MeV}$ for ${ }^{56} \mathrm{Fe}$ ), the damage energy is not sensitive to PKA energy. For PKA with atomic number and atomic mass close to those of target atom, the maximum damage energy is $E_{a}^{\max } \approx 573 Z^{5 / 3} A^{1 / 2} \mathrm{eV}$.

The relativistic effect on recoil PKA energy of discrete reactions depends on reaction type, target nucleus, excitation level, emission angle, and incident neutron energy [3]. For incident neutron energy below $20 \mathrm{MeV}$, the relativistic correction is within 1\%. Except for the extension of the range of PKA energy, the relativistic effect has limited influence on neutron-induced atomic displacement in both fission and fusion reactors. However, for incident neutron energy up to $200 \mathrm{MeV}$ or even $800 \mathrm{MeV}$ for spallation neutron sources, the relativistic kinematics should be used to calculate the PKA energy from nuclear reactions. $R / C-1(\%) \approx 0.05 E_{\text {in }} / \mathrm{MeV}$ for neutron elastic scattering for nuclei from ${ }^{56} \mathrm{Fe}$ to ${ }^{238} \mathrm{U}$. The relativistic effect is less important for $(\mathrm{n}, \alpha)$ and $(\mathrm{n}, \mathrm{t})$ reactions.

Because the damage energy (or the partition function) depends much on the atomic numbers and atomic masses of both PKA and target atom, the relativistic effect on damage energy varies from nucleus to nucleus. For nuclei lighter than ${ }^{56} \mathrm{Fe}$, the relativistic corrections are generally within $2 \%$ for incident neutron energy up to $200 \mathrm{MeV}$ because of the "saturation" of damage energy. However, we remark again that the current damage theory cannot be applied for PKA energy higher than $24.9 A_{R} Z_{R}^{4 / 3} \mathrm{keV}$.

\section{References}

[1] C. Lemaignan, Handbook of Nuclear Engineering, (Springer, 2010) 543-642

[2] S. Chen and D. Bernard, Nucl. Instrum. Methods Phys. Res. Sect. B, 440, 107-113 (2019)

[3] S. Chen and D. Bernard, J. Nucl. Mater., 522, 236-245 (2019)

[4] A. Santamarina et al., JEFF Report 22, NEA No. 6807, (2009)

[5] S. Chen, D. Bernard, and L. Buiron, Nucl. Eng. Des., 346, 85-96 (2019)

[6] S. Chen et al.,, Nucl. Instrum. Methods Phys. Res. Sect. B, 456, 120-132 (2019)

[7] J. Lindhard, et al., Mat. Fys. Medd. Dan. Vid. Selsk. 33(10), 1-42 (1963)

[8] M. J. Norgett, M. T. Robinson, and I. M. Torrens, Nucl. Eng. Des. 33(1), 50-54 (1975) 